\title{
An Economic Analysis of Critical Care Nurse Resourcing Following the Uptake of Ready-to- Administer Noradrenaline for Hypotensive Shock in Adults in England
}

\author{
Edward Griffin (D) - Oliver Tweedie · Justin Kirk-Bayley
}

Received: October 14, 2021 / Accepted: November 17, 2021 / Published online: December 7, 2021

(C) The Author(s) 2021

\begin{abstract}
Introduction: Ready-to-administer formulations for intravenous administration of noradrenaline are now broadly recommended and predicted to reduce pressure on critical care nursing. This analysis sought to quantify the nurse resource released from national level transition.

Methods: The annual number of noradrenaline support days for hypotensive shock was determined and the administration of noradrenaline was simulated over $24 \mathrm{~h}$ using a decision tree. A 'best-practice' ready-to-administer strategy (RtA) of volumetrically pumped noradrenaline was compared to a 'nil uptake' strategy (AfC) of bedside prepared solution delivered either volumetrically or using a double syringe pump. A mix of noradrenaline concentrations, flow rates, product sizes, and preferences for ampoule pooling, preparation volume, and sterility were included. The consumption of nurse days and product units was then projected over 1 year for
\end{abstract}

E. Griffin

Ed Griffin Ltd, Exeter, UK

O. Tweedie $(\varangle)$

Sintetica Ltd, 30th Floor, 40 Bank Street, Canary

Wharf, London E14 5NR, UK

e-mail: Oliver.Tweedie@sintetica.co.uk

J. Kirk-Bayley

Royal Surrey NHS Foundation Trust, Guildford, UK a population of adults in critical care in England.

Results: Noradrenaline was administered over 231,011 days per year across 4123 critical care beds in England. Implementing a transition from AfC to RtA strategies on this scale released 35,791 nurse days or 176 whole-time nurse equivalents at 50/50 NHS band 5 and 6 , a monetised release of $£ 11.6$ million. There was an increase in drug acquisition cost of $£ 2.1$ million using the licensed commercial product Sinora ${ }^{\circledR}$. Annual net monetary benefit was $+£ 9.5$ million, or $+£ 65,961$ per critical care unit (CCU) of 29 beds, equivalent to one nurse released per unit for patient care.

Conclusions: This modelling of ready-to-administer noradrenaline with volumetric delivery quantifies and bears out the recommendations of the Lord Carter review, the Royal Pharmaceutical Society, and the NHS Specialist Pharmacy Service in their encouragement of ready-to-administer formulations for safe and resource-effective critical care.

Keywords: Budget impact; Hypotensive shock; Medication safety; Noradrenaline; Nursing; Ready-to-administer; Resource-saving 


\section{Key Summary Points}

Why carry out this study?

Ready-to-administer intravenous products are now recommended for the critical care setting but some NHS trusts are yet to implement. Quantitative evidence may assist the decision maker.

What was learned from the study?

A transition to ready-to-administer noradrenaline releases nurse time for patient care, drug budget uplift is moderate, and net monetary benefit is favourable.

A ready-to-administer strategy is less wasteful of noradrenaline.

\section{INTRODUCTION}

Bedside preparation of noradrenaline from concentrate remains common practice in critical care, but ready-to-administer (RtA) formulations which are pre-mixed to standardised concentrations are available and encouraged. Lord Carter's most recent national report, looking at intravenous (IV) injectables, assesses that this aspect of delivery often exceeds $10 \mathrm{~min}$, which is time that could otherwise be spent on direct patient care. It offers ready-to-administer formulations as a way to release nurses and increase the safety and the transparency of quality assurance for patients [1]. By way of context, NHS England and NHS Improvement, with Health Education England, have issued new guidance on workforce models for hospitals during COVID-19, including staffing critical care services with decreasing nurse ratios as they operate beyond their capacity [2]. However, the reported benefit of ready-toadminister preparations extends beyond resource saving. Mixing of concentrated formulations is usually undertaken in an environment with clinical distractions and other pressures, increasing the likelihood of error. The NHS
Specialist Pharmacy Service demonstrates that procurement of licensed ready-to-administer product with marketing authorisation is the least risk-associated option across all categories assessed, and the best use of pharmacy skills to support to clinical areas [3]. The Royal Pharmaceutical Society recommends that the manipulation of medicines outside pharmacy should be minimised, and states that injectable medicines should be presented as prefilled syringes or other 'ready-to-administer' preparations wherever possible [4].

The objective of this research is to provide quantitative evidence to assist healthcare decision makers plan for RtA noradrenaline. This analysis considers a range of relevant clinical variables about this objective, but its scope is limited to the evaluation of nurse and product resource use. Specifically, it does not include health outcomes as impacted by harm resultant from administration error rates, which may be improved with centrally pre-prepared solutions $[5,6]$. This article is based on previously conducted studies and does not contain any new studies with human participants or animals performed by any of the authors.

\section{METHODS}

A budget impact model developed to compare competing user-defined local strategies for the administration of noradrenaline was adapted for the national health service level. It was parameterised to assess a strategy simulating a starting or nil uptake position (administration from concentrate-AfC) and a strategy representing full transition or best practice (ready-toadminister-RtA). The AfC strategy was assimilated on the basis of expert opinion to reflect a 2019 pre-COVID-19 real-world picture of the delivery of noradrenaline when prepared from concentrate [7]. Solution is prepared by the critical care nurse at the bedside and is delivered by double syringe pump in $80 \%$ of cases and volumetric pump in $20 \%$ of cases. The best practice strategy was an implementation of ready-to-administer noradrenaline delivered by volumetric pump in all cases. The comparison therefore considered alternative approaches in 
Table 1 Model parameters and settings

Preparation of solution for infusion

Proportion concentrated noradrenaline prepared at bedside

Proportion of concentrated noradrenaline prepared aseptically

Proportion of preparation and pump set-up by band 5 nurse

Proportion of preparation and pump set-up by band 6 nurse

Noradrenaline waste, single or volumetric pump changeover (ml)

Noradrenaline waste, double pump changeover $(\mathrm{ml})$

Maximum preparation sterility period ( $h$ )

Staff time on preparation tasks ( $\mathrm{min}$ )

1. Prepare, check, label preparation

AfC

RtA

2. Second nurse checking

AfC

3

RtA

3. Pump set-up or prep changeover

Single or volumetric

Double

Unit costs of consumables and resources

$4 \mathrm{mg}$ in $4 \mathrm{ml}$ noradrenaline ampoule

$8 \mathrm{mg}$ in $8 \mathrm{ml}$ noradrenaline ampoule

$£ 3.20[13]$

$4 \mathrm{mg}$ in $50 \mathrm{ml}$ vial ready-to-administer noradrenaline (Sinora ${ }^{\circledR}$ )

$8 \mathrm{mg}$ in $50 \mathrm{ml}$ vial ready-to-administer noradrenaline (Sinora ${ }^{\circledR}$ )

$£ 7.37[18]$

$5 \%$ dextrose or $0.9 \%$ saline, $100 \mathrm{ml}$

$£ 1.47[14]$

Nurse band 5 per working hour

Nurse band 6 per working hour

Clinical preferences for formulation and administration

AfC

Formulation: concentrated

Formulation: ready-to-administer

Requirement low. $0.1 \mu \mathrm{g} / \mathrm{kg} / \mathrm{min}$ noradrenaline*

Requirement high. $0.2 \mu \mathrm{g} / \mathrm{kg} / \mathrm{min}$ noradrenaline*

Requirement very high. $0.4 \mu \mathrm{g} / \mathrm{kg} / \mathrm{min}$ noradrenaline*

Flow rate low. $2.8 \mathrm{ml} / \mathrm{h}^{*}$

$0 \%$

$0 \%$

Flow rate medium. $5.6 \mathrm{ml} / \mathrm{h}^{*}$

Flow rate high. $11.3 \mathrm{ml} / \mathrm{h}^{*}$

Pooling of $4 \mathrm{mg}$ ampoules to $8 \mathrm{mg}$ solution*

Pooling of $4 \mathrm{mg}$ ampoules to $16 \mathrm{mg}$ or $32 \mathrm{mg}$ solution*

Proportion double pumped*

0\%

Proportion single or volumetrically pumped*

Preparation volume small. $50 \mathrm{ml}^{*}$

$100 \%$

Preparation volume medium. $100 \mathrm{ml}^{*}$

N/A

$A f C$ administered from concentrate (strategy), RtA ready-to-administer (strategy), $N / A$ not applicable

*Based on clinical expert opinion 
both the formation and delivery of noradrenaline. Model parameters and settings are listed in Table 1.

A decision tree, constructed in Microsoft $365^{\circledR}$ Excel $^{\circledR}$, was considered appropriate versus more complex methods given the absence of temporal determinants of outcomes (e.g. occurrence of clinical events). Chance nodes facilitated strategy differences for a population of adults admitted to NHS critical care in England and requiring noradrenaline vasopressor support for hypotensive shock. The critical care unit (CCU) effectively comprised the intensive care unit (ICU, level 3) and high dependency unit (HDU, level 2). Chance nodes also facilitated relevant clinical and procedural preferences common to both strategies about which uncertainty at national level should be evaluated (e.g. sterility protocol. See Fig. 1 legend). The analysis took the health system payor perspective and evaluated a time horizon of 1 year, a projection of 24 -h resource consumption based on the annual number of days of noradrenaline vasopressor support for hypotensive shock. Support days were annual noradrenaline admissions for septic shock in England, adjusted upward by one-third to include undifferentiated cases, multiplied by the mean length of stay for septic shock [8-10].

Modelled outcomes were annual nurse resource (primary outcome); cost of nurse resource; episodes of noradrenaline preparation; volume of noradrenaline acquired but not infused (waste); product units of noradrenaline and dextrose or saline; cost of products; and cost of nurse resource less cost of drug acquisition-the net monetary benefit (NMB). For those cases where pharmacy technicians undertake noradrenaline preparation, the resource was treated as critical care nursing, itself divided into either drug preparation or pump management. Drug preparation included a first nurse drawing-up noradrenaline, labelling and checking the syringe or infusion bag, and a second nurse performing checks. Pump management comprised the tasks of pump setup and reloading of preparations, assumed equal in time. Task times differed by formulation and pumping approach. The extent and nature of nurse tasks, and their average duration, were estimated by survey between ten nursing and medical clinical experts from different NHS critical care providers, except for the time to prepare solution from concentrate, which was sourced from the Lord Carter report $[1,7]$.

Resources were monetised in GBP using the 2020 cost year, inflating as required using the NHS Cost Inflation Index [11]. The hourly cost of hospital nurse day ( $7.5 \mathrm{~h}$ and 50/50 band 5/6) was sourced from the PSSRU Unit Costs of Health and Social Care handbook 2019 and inflated by 1 year [12]. Whole time equivalents (WTEs) were calculated by dividing annual nursing cost by the band-weighted annual salary (50\% NHS band 5, 50\% band 6). Lowest available prices for non-proprietary products were sourced from the DHSC national eMIT average pricing tool or the British National Formulary [13, 14]. Ready-to-administer noradrenaline was either $4 \mathrm{mg}$ in $50 \mathrm{ml}$ or $8 \mathrm{mg}$ in $50 \mathrm{ml} \mathrm{Sinora}{ }^{\circledR}[15,16]$. Their prices were obtained from the manufacturer $[17,18]$. Product unit size was optimally matched to population requirement, except for the practice of ampoule pooling. All patients were assumed to be $75 \mathrm{~kg}$ and infusion bags were $100 \mathrm{ml}$.

Uncertainty was explored using both fixed variation univariate sensitivity analysis (parameter uncertainty) and alternative scenario analysis (structural uncertainty). Both measured the impact of variation on NMB at CCU level.

\section{RESULTS}

\section{Service Size and Nurse Burden}

The model estimated 30,396 annual adult admissions and 231,011 inpatient days requiring vasopressor support with noradrenaline (Table 2). Using a strategy of noradrenaline from concentrate (AfC) the associated nurse resource requirement (primary outcome) was 41,952 working days, two-thirds drug preparation and one-third pump management (Table 3). Using a strategy of ready-to-administer noradrenaline delivered by volumetric pump (RtA), the nurse resource was reduced by $85 \%$ to 6160 working days, equivalent to a release of 


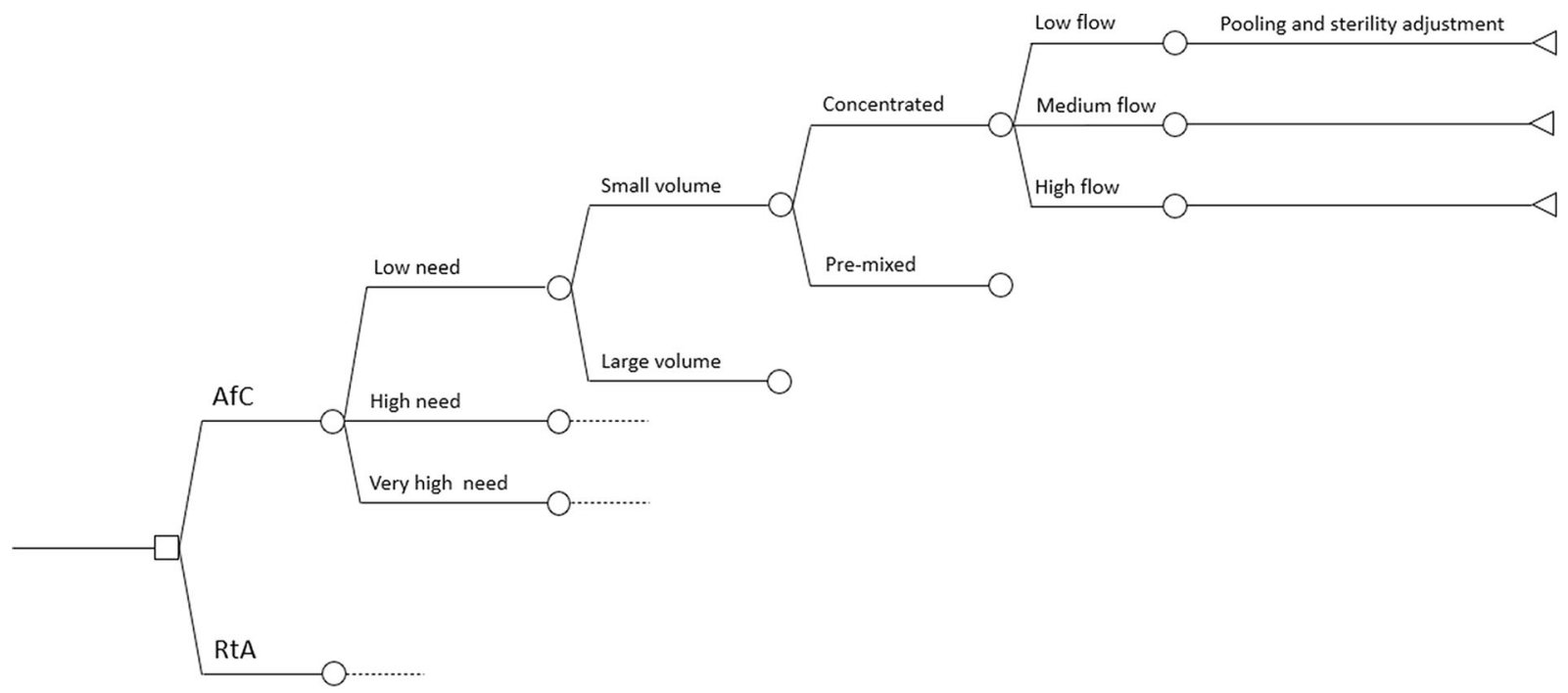

Fig. 1 Simplified decision tree. AfC Administered from concentrate (strategy), RtA ready-to-administer (strategy). The square is the decision node and represents the strategy option. Chance nodes facilitate the choices for population and health system preferences. The first chance node allows a distribution to be set for the noradrenaline requirement across the modelled population. Levels are $0.1 \mu \mathrm{g} / \mathrm{kg} / \mathrm{min}$ (low), $0.2 \mu \mathrm{g} / \mathrm{kg} / \mathrm{min}$ (high), or $0.4 \mu \mathrm{g} / \mathrm{kg} /$

176.3 WTEs across England (109 and 67 WTEs for drug preparation and pump management respectively), or 1.2 WTEs for a CCU of 29 beds.

\section{Noradrenaline Preparation Frequency and Waste}

Both noradrenaline consumption and preparation frequency were higher using a strategy of noradrenaline from concentrate (AfC) versus the RtA strategy: 3.6 preparations per patient per day and $33.6 \mathrm{mg}$ of noradrenaline, versus 3.0 and $25.2 \mathrm{mg}$ (Table 4). This is explained by the difference between strategies in their susceptibility to waste. There was an additional $4.8 \mathrm{mg}$ daily loss from discarding of non-depleted vessels consequent to protocol-driven sterility changes (24-h mandatory change in the base case). These occurrences are more likely with the higher volume $100 \mathrm{ml}$ preparations used for volumetric pumping in the AfC strategy, and less likely in CCUs with longer sterility periods. For example, increasing the sterility period from 24 to $48 \mathrm{~h}$ reduces the mean daily min (very high). The second chance node allows the adjustment of preparation volume, which is $50 \mathrm{ml}$ for RtA vials but either $50 \mathrm{ml}$ or $100 \mathrm{ml}$ for dilution. The third chance node facilitates the preference for formulation: concentrate, ready-to-administer, or a combination. The final chance node dictates the flow rate preference and allows a distribution across the rates $2.8 \mathrm{ml} / \mathrm{h}$ (low), $5.6 \mathrm{ml} / \mathrm{h}$ (standard), and $11.3 \mathrm{ml} / \mathrm{h}$ (high)

preparation frequency from 3.6 to 3.1 , but the impact diminishes only marginally to 3.0 in CCUs adopting a 72-h sterility protocol. The average daily waste from loss associated with changing double-pump syringes (AfC strategy only) was $21.6 \mathrm{ml}$ of solution. Both sources have waste thresholds about which outcomes are sensitive. For example, changeover loss of below $6 \mathrm{ml}$ per syringe reload decreases the daily mean preparation frequency by 0.6 .

\section{Resource Cost and Net Monetary Benefit}

The amount of product needed per patient per day for the AfC strategy was 5.9 units of the $4 \mathrm{mg}$ ampoule and 1.3 units of the $8 \mathrm{mg}$ ampoule, compared to 0.3 units of the $4 \mathrm{mg}$ in $50 \mathrm{ml}$ syringe and 3.0 units of the $8 \mathrm{mg}$ in $50 \mathrm{ml}$ syringe (Table 4). Ampoule pooling in fact reduced the overall acquisition cost of concentrated noradrenaline since the small unit is sourced at less than half the price of the larger unit. Totalled product acquisition costs were $£ 2.4$ million and £5.7 million for AfC and RtA 
Table 2 Estimation of the adult noradrenaline service size in England

\section{Parameter}

Critical care beds in England (intensive care and high dependency)

$4,123[16]$

Total critical care admissions for shock per year with noradrenaline support

Mean days of noradrenaline support per admission

Total patient days of noradrenaline support per year

231,011

Table 3 Cumulative task time spent by critical care nurses preparing and delivering noradrenaline (7.5 h working days)

\begin{tabular}{llll}
\hline Nurse task & AfC & RtA & Increment (change) \\
\hline Drug preparation and checking (syringe/infusion bag) & 26,797 & 4,620 & $-22,177(83 \%)$ \\
Pump set-up and subsequent changeovers & 15,154 & 1,540 & $-13,614(90 \%)$ \\
Total & 41,952 & 6,160 & $-35,791(85 \%)$ \\
Total whole-time-equivalents (WTEs) & 206.7 & 30.3 & 176.3 \\
\hline
\end{tabular}

Table 4 Noradrenaline preparation frequency and consumption, per $24 \mathrm{~h}$

\begin{tabular}{|c|c|c|c|}
\hline Item & AfC & RtA & Increment \\
\hline Episodes of noradrenaline preparation & 3.6 & 3.0 & -0.6 \\
\hline Volume of solution lost to line priming at changeover $(\mathrm{ml})$ & 21.6 & 0.0 & -21.6 \\
\hline Total volume of noradrenaline consumed (mg) & 33.6 & 25.2 & -8.4 \\
\hline Volume of noradrenaline lost due to sterility changes (mg) & 7.3 & 2.5 & -4.8 \\
\hline Product units of $4 \mathrm{mg}$ noradrenaline & 5.9 & 0.3 & -5.6 \\
\hline Product units of $8 \mathrm{mg}$ noradrenaline & 1.3 & 3.0 & 1.7 \\
\hline Proportion of ready-to-administer noradrenaline at $4 \mathrm{mg}$ & $0 \%$ & $9 \%$ & - \\
\hline Proportion of ready-to-administer noradrenaline at $8 \mathrm{mg}$ & $0 \%$ & $91 \%$ & - \\
\hline
\end{tabular}

strategies respectively, representing a national budget uplift of $£ 3.3$ million (Table 5). This compares to an $£ 11.6$ million monetisation of the estimated 176 WTE country-wide nurse release. Combining product acquisition and nurse resourcing, the net monetary benefit was $+£ 9.5$ million per year for England (145 WTEs), or $+£ 65,961$ for a CCU of 29 beds (1.0 WTE).

\section{Uncertainty}

The univariate sensitivity analysis of 22 input parameters (Fig. 2) identified two parameters for which CCU NMB deviated by a proportion higher than the $20 \%$ input variation: the sterility period, and the proportion of noradrenaline double pumped. Testing of structural assumptions in the scenario analysis highlighted the impact noradrenaline delivery has on NMB beyond the impact of formulation 
Table 5 Cost and net costs for all adult critical care episodes of noradrenaline support of hypotensive shock in England

\begin{tabular}{llll}
\hline Item & AfC & RtA & Increment \\
\hline Cost of noradrenaline from concentrate & $£ 2,374,281$ & $£ 0$ & $-£ 2,374,281$ \\
Cost of ready-to-administer noradrenaline & $£ 0$ & $£ 5,655,161$ & $£ 5,655,161$ \\
Cost of noradrenaline, any formulation & $£ 2,374,281$ & $£ 5,655,161$ & $£ 3,280,881$ \\
Cost of dextrose or saline for dilution & $£ 1,222,513$ & $£ 0$ & $-£ 1,222,513$ \\
Total cost of noradrenaline and dextrose & $£ 8,652,692$ & $£ 5,655,161$ & $-£ 2,997,530$ \\
Cost of drug preparation tasks & $£ 8,652,692$ & $£ 1,491,843$ & $-£ 7,160,848$ \\
Cost of pump preparation and reload tasks & $£ 4,893,246$ & $£ 497,281$ & $-£ 4,395,965$ \\
Total cost of nursing time noradrenaline tasking & $£ 13,545,938$ & $£ 1,989,124$ & $-£ 11,556,813$ \\
Net monetary benefit, England (per year) & & & $£ 9,498,445$ \\
Net monetary benefit, per 29 bed CCU (per year) & & & $£ 65,961$ \\
Net monetary benefit, per CCU bed (per year) & & & $£ 2,304$ \\
\hline
\end{tabular}

(Table 6). The scenario removing the trend to volumetric pumping [1] reduced CCU NMB by $39 \%$. A threshold analysis of a scenario eliminating double pumping (all volumetric) found 2.4 fewer minutes (21\% less time) to mix a preparation from concentrate reduced $\mathrm{NMB}$ to below zero.

\section{DISCUSSION}

The Lord Carter report 'Transforming NHS Pharmacy Aseptic Services in England' predicts that ready-to-administer preparations will release critical care nurses to patient care [1]. In respect to noradrenaline we quantified this as 176 whole time nurse equivalents-realisable time that can be put to all the associated and unmeasurable benefits for patient wellbeing and enhanced care. We demonstrate that waste is an important differentiating factor and is reduced by ready-to-administer products under plausible clinical preferences and unit protocol. We also predict new pharmacy budget pressure from the acquisition of RtA products, but in a context of favourable net monetary benefit.

This is the first analysis of critical care resourcing for transition to ready-to-administer noradrenaline known to the authors. By incorporating country-wide variation in noradrenaline concentration, flow rate, ampoule pooling, and delivery method, we have included the impact waste has on resources and costs, which is demonstrated to be relevant. Further, individual activities undertaken by the critical care nurse are imputed at the microlevel, so that their impact on outcomes can be understood. However, the findings may be conservative. There is a body of evidence linking bedside preparation with a higher rate of error compared to centrally pre-prepared solutions and this has not been considered here [5, 6, 19-21]. We have assumed a position of 'no difference' given the scarcity of informative evidence. Valentin and colleagues conducted a large multinational prospective study of error rates in the administration of parenteral drugs, and they report specifically for vasopressors and catecholamines, but theirs is not a comparative study [6]. Dehmel and colleagues conducted a preliminary laboratory study of CCU and centrally prepared samples, expressing their findings as deviations in concentration conformity but they did not report a link to harm [5]. New comparable evidence of harm from administration error is required before a cost-effectiveness analysis is feasible, and this is a recommended area for future research. Other limitations 


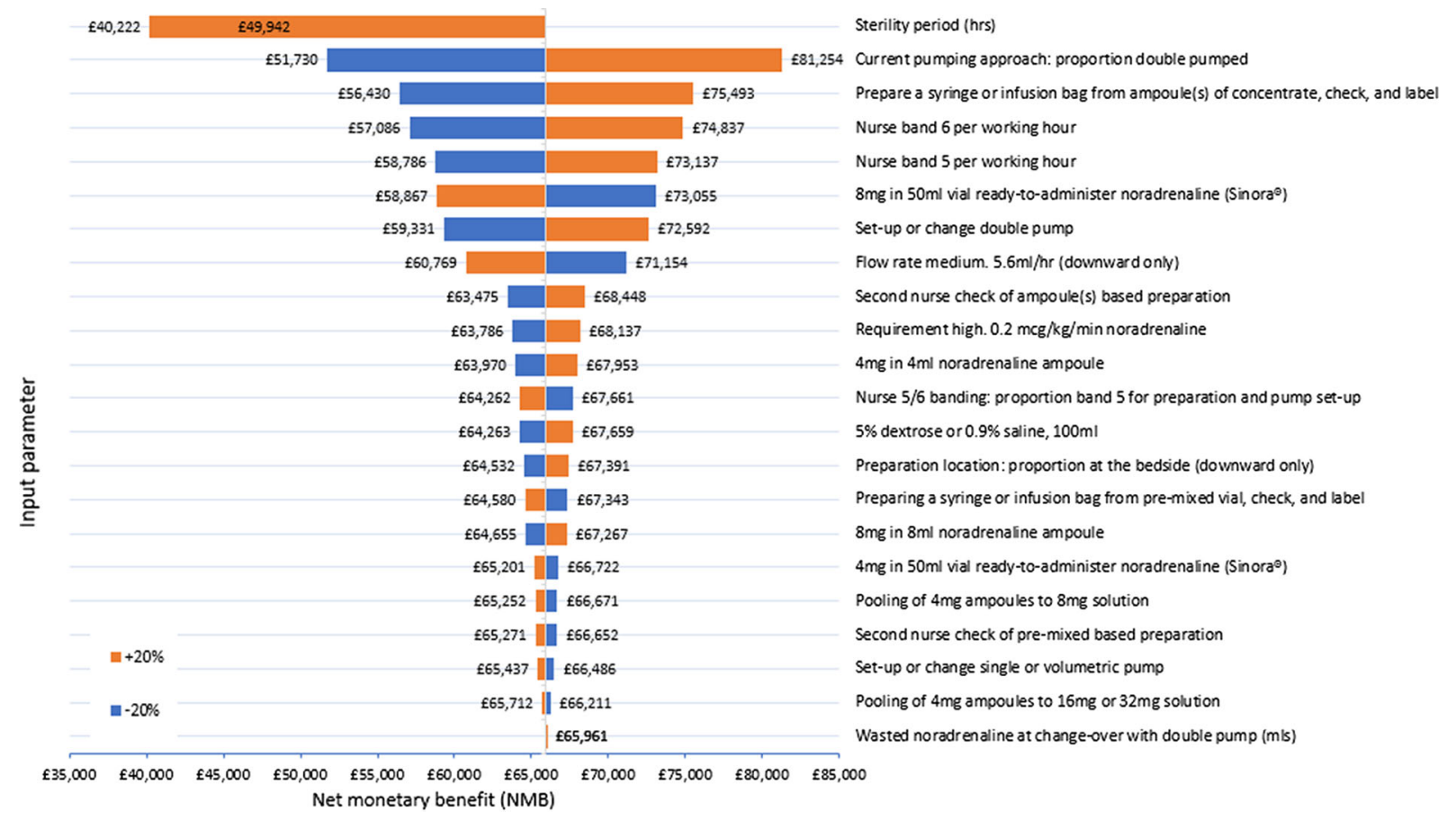

Fig. 2 One-way sensitivity analysis of NMB for towards 20\% input parameter variation (average CCU of 29 beds)

concern uncertainty around input parameters, with reliance on expert opinion of the current national picture of clinical preference, as well as the duration of most tasks. The estimate of the size of the national service was necessarily an approximation. Sensitivity analysis showed some robustness in the findings but also highlighted the precarious decision problem. A transition to volumetric pumping will in itself release nurses. A threshold analysis of task time when both strategies used volumetric pumping showed incremental outcomes to be sensitive to time-efficient tasking. It is important to stress that the headline benefits we report require a transition to volumetric pumping as well as the ready-to-administer formulation.

Our findings are indicative of unmet opportunity at the national level, but we recommend bespoke modelling at the local level given the importance for outcomes of clinical preference and protocol. So too, these results may not be generalisable to other healthcare systems.

\section{CONCLUSION}

Implementing at scale ready-to-administer noradrenaline delivered by a volumetric pump will release nurses for patient care. This finding adds to the encouragement given in the Lord Carter Report and recent guidelines from the Royal Pharmaceutical Society and the NHS Specialist Pharmacy Service.

\section{ACKNOWLEDGEMENTS}

Funding. Sponsorship of this study and Rapid Service and Open Access Fees were funded by Sintetica Ltd (London, UK).

Authorship. All named authors meet the International Committee of Medical Journal Editors (ICMJE) criteria for authorship for this article, take responsibility for the integrity of the work as a whole, and have given their approval for this version to be published. 


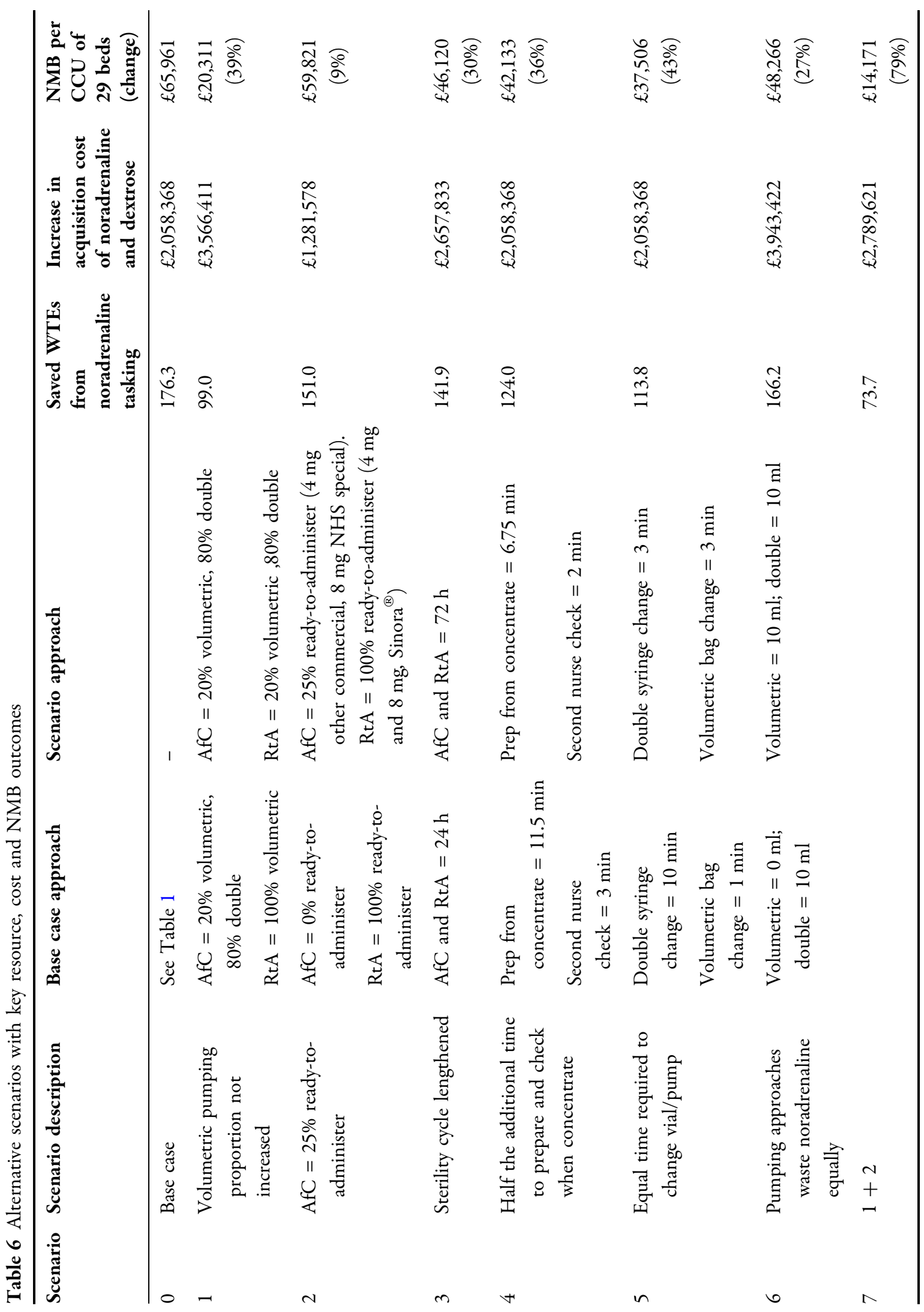




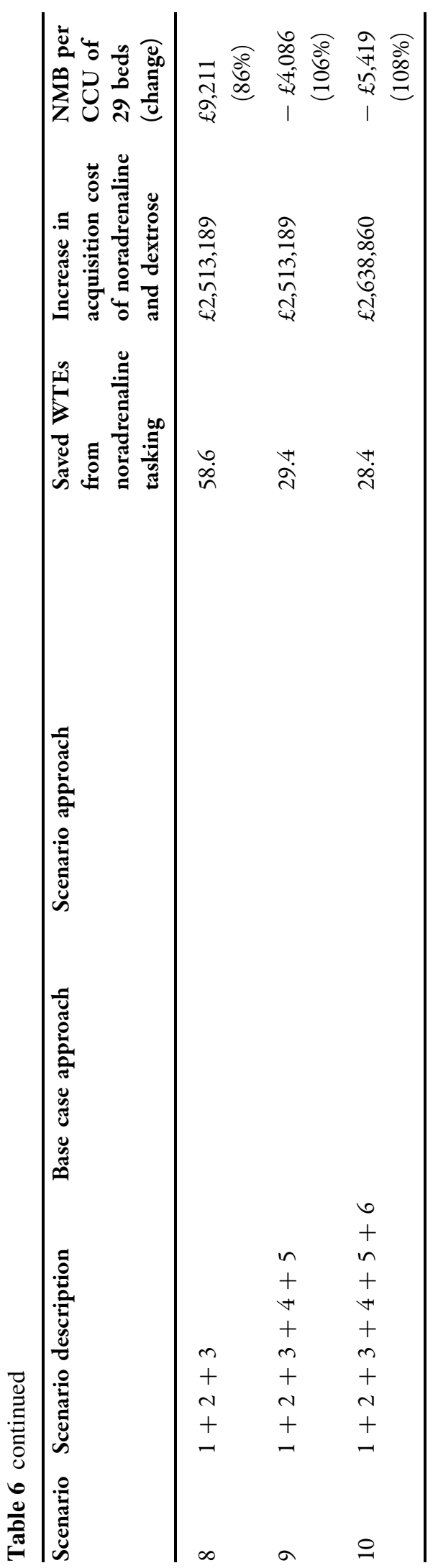

Author Contributions. EG designed and performed the analysis, and drafted the manuscript; OT conceptualised the decision problem; JK-B provided clinical insight and helped draft the manuscript.

Disclosures. Edward Griffin and Justin KirkBayley have received payment as consultants to Sintetica Ltd. Oliver Tweedie is an employee of Sintetica Ltd.

Compliance and Ethics Guidelines. This article is based on previously conducted studies and does not contain any new studies with human participants or animals performed by any of the authors.

Data Availability. Data sharing is not applicable to this article as no datasets were generated or analysed during the current study.

Open Access. This article is licensed under a Creative Commons Attribution-NonCommercial 4.0 International License, which permits any non-commercial use, sharing, adaptation, distribution and reproduction in any medium or format, as long as you give appropriate credit to the original author(s) and the source, provide a link to the Creative Commons licence, and indicate if changes were made. The images or other third party material in this article are included in the article's Creative Commons licence, unless indicated otherwise in a credit line to the material. If material is not included in the article's Creative Commons licence and your intended use is not permitted by statutory regulation or exceeds the permitted use, you will need to obtain permission directly from the copyright holder. To view a copy of this licence, visit http:// creativecommons.org/licenses/by-nc/4.0/.

\section{REFERENCES}

1. Coles P. Transforming NHS pharmacy aseptic services in England: a national report for the Department of Health and Social Care by Lord Carter of Coles. London: Department of Health and Social Care; 2020. 
2. NHS England. Advice on acute sector workforce models during COVID-19, Annex 1 (NHS England, NHS Improvement, Health Education England). 2020. URL: https://www.england.nhs.uk/ coronavirus/wp-content/uploads/sites/52/2020/12/ C0833_advice-on-acute-sector-workforce-modelsduring-COVID_with-apps_10dec.pdf. Accessed March 2021.

3. Allen I. Options for the provision of ready to administer IVs to support clinical areas-COVID19. NHS Specialist Pharmacy Service. 2020.

4. Royal Pharmaceutical Society. Professional guidance on the safe and secure handling of medicines RPS. 2018. URL: https://www.rpharms.com/recog nition/setting-professional-standards/safe-and-secu re-handling-of-medicines/professional-guidance-on -the-safe-and-secure-handling-of-medicines. Accessed November 2020.

5. Dehmel C, Braune SA, Kreymann G, et al. Do centrally pre-prepared solutions achieve more reliable drug concentrations than solutions prepared on the ward? Intensive Care Med. 2011;37(8):1311-6. https://doi.org/10.1007/s00134-011-2230-4.

6. Valentin A, Capuzzo M, Guidet B, et al. Errors in administration of parenteral drugs in intensive care units: multinational prospective study. BMJ. 2009;338:b814. https://doi.org/10.1136/bmj.b814.

7. Tweedie O. Medical Director, Data on file Sintetica Ltd. 2021.

8. Andersson FL, Palencia R, Kjølbye AL. PHP90 - Costs Of Septic Shock In England, Wales And Northern Ireland In 2012. Value in Health. 2015;18(7):A530.

9. Office of National Statistics (ONS). Estimates of the population for the UK, England and Wales, Scotland and Northern Ireland. People, Population and Community. 2020 (Mid-Year Population Estimates, UK, June 2020). URL: https://www.ons.gov.uk/ peoplepopulationandcommunity/populationandmi gration/populationestimates/datasets/populationes timatesforukenglandandwalesscotlandandnorther nireland. Accessed Nov 2020.

10. De Backer D, Biston P, Devriendt J, et al. Comparison of dopamine and norepinephrine in the treatment of shock. N Engl J Med. 2010;362(9): 779-89.
11. Personal Social Services Research Unit (PSSRU University of Kent). NHS Cost Inflation Index (NHSCII). PSSRU. 2019. URL: https://www.pssru.ac. uk/pub/uc/uc2019/NHS-Cost-Inflation-Index.docx. Accessed Nov 2020.

12. Curtis L, Burns, A. Unit costs of health and social care 2019. PSSRU, University of Kent, Book; Unit Costs of Health and Social Care, vol. 176; 2019.

13. GOV.UK. Drugs and pharmaceutical electronic market information tool (eMIT). Department of Health and Social Care. 2021. URL: https://www. gov.uk/government/publications/drugs-and-pharm aceutical-electronic-market-information-emit. Accessed Nov 2020.

14. National Institute for Health and Care Excellence. British National Formulary. BNF. 2021. URL: https://bnf.nice.org.uk/. Accessed Nov 2020.

15. Sintetica Ltd. Summary of Product Characteristics Sinora $0.08 \mathrm{mg} / \mathrm{ml}$. EMC. 2020.

16. Sintetica Ltd. Summary of Product Characteristics Sinora $0.16 \mathrm{mg} / \mathrm{ml}$. EMC. 2020.

17. NHS CMU. $4 \mathrm{mg}$ in $50 \mathrm{ml}$ Sinora unit price; NHS National Framework Agreement for the Supply of Generic Medicines. 2021. URL: https://www.england. nhs.uk/medicines-2/commercial-medicines/commer cial-medicines-unit/. Accessed October 2021.

18. Sintetica Ltd. $8 \mathrm{mg}$ in $50 \mathrm{ml}$ Sinora unit price; supplied by manufacturer. 2021.

19. Stucki C, Sautter AM, Wolff A, Fleury-Souverain S, Bonnabry P. Accuracy of preparation of i.v. medication syringes for anesthesiology. Am J Health Syst Pharm. 2013;70(2):137-42.

20. Hedlund N, Beer I, Hoppe-Tichy T, Trbovich P. Systematic evidence review of rates and burden of harm of intravenous admixture drug preparation errors in healthcare settings. BMJ Open. 2017;7(12): e015912.

21. Cousins DH, Sabatier B, Begue D, Schmitt C, Hoppe-Tichy T. Medication errors in intravenous drug preparation and administration: a multicentre audit in the UK, Germany and France. Qual Saf Health Care. 2005;14(3):190-5. 